\title{
De la evaluación tradicional a una nueva evaluación basada en competencias
}

\section{From Traditional Assessment to the New Competency-Based Assessment}

\author{
Omar Mejía Pérez ${ }^{1}$ \\ Universidad Autónoma de Fresnillo \\ Cuernavaca, Morelos, México \\ klvita@hotmail.com
}

Recibido 21 de julio de 2011 • Aceptado 26 de agosto de 2011

\begin{abstract}
Resumen. La evaluación escolar - de los alumnos- a lo largo del tiempo ha sido un elemento que ha intentado estimar el esfuerzo, capacidad y aprendizaje escolar. No obstante, en sus inicios, sus formas eran mediciones parciales de la evolución de la conducta con respecto a un comportamiento deseable. Más tarde, la evaluación fue sufriendo cambios que beneficiaron los procesos de valoración y enjuiciamiento del desempeño académico de los aprendices y su propio aprendizaje. En la actualidad, las exigencias de las sociedades contemporáneas son vastas y múltiples; los educandos no requieren exclusivamente aprender conocimientos: es necesario ayudarles a desarrollar habilidades, valores y actitudes. La educación posmoderna exige el desarrollo de diversas facultades y capacidades que les permitan a los individuos evolucionar de manera integral. Por tanto, la evaluación, en ese sentido, debe responder a tales necesidades de enjuiciamiento didácticas, valorando ética, técnica y fehacientemente el desarrollo evolutivo de las competencias de los educandos y, así, poder emitir juicios cualitativos y cuantitativos de manera más justa y objetiva. Este proyecto de disertación es el resultado de la revisión bibliográfica de varios autores y el trabajo cotidiano docente en los Centros de Educación Media Superior a Distancia del Estado de Morelos, México.
\end{abstract}

Palabras claves. Evaluación, tradicionalismo, competencias.

Abstract. The evaluation of school students has always attempted to estimate the effort, abilities and learning of students. However, at the beginning, evaluation consisted in measuring the progress of the student's behavior compared to a desirable behavior. Later on, different changes in evaluation benefited the processes addressed to assessing the academic achievement of students and learning itself. Today, the demands of the contemporary society are vast and numerous: students not only require knowledge, they need to develop skills, values and postures. Postmodern education requires individuals to develop different talents and competencies to grow in every way. Therefore, evaluation should respond to such needs promoting an ethical, technical, reliable assessment of the student's competencies, thus providing more fair and objective, qualitative and quantitative judgments. This dissertation project is the result of a literature review of several authors and the daily work of teachers in the Centros de Educación Media Superior a Distancia [High School Distance Centers] of Morelos, Mexico.

Keywords. Assessment, traditionalism, competencies.

\footnotetext{
Maestro en Educación, por la Universidad Autónoma de Fresnillo, México. Doctorando por el Centro de Estudios Superiores de México en coordinación con la Universidad Autónoma de Fresnillo en el Doctorado en Educación. Docente en la Universidad Autónoma de Morelos, en la Universidad del Valle de México y en el Colegio de Bachilleres del Estado de Morelos. Ha laborado para el Instituto de Educación Básica del Estado de Morelos, el Centro Morelense de las Artes, la Universidad de la Cuenca de Morelos y el Centro de Estudios Superiores de México. Actualmente está por concluir su trabajo de tesis doctoral.
} 


\section{A manera de introducción}

El proceso de evaluación dentro del ámbito educativo ha tenido a lo largo de su historia distintas formas de apreciación, distintos usos, metodologías de aplicación y objetivos. Actualmente, desde los nuevos enfoques educativos, la evaluación ha intentado resurgir como un proceso capaz de replantear la orientación de la educación en sí, mediante el reciclaje de los resultados dentro de los momentos de planeación y desarrollo de la intervención didáctica de manera más objetiva; sin embargo, el desconocimiento de su función real, sus dimensiones y fines pueden entorpecer el propio desarrollo didáctico y determinación de resultados en materia educativa.

La evaluación del progreso escolar de los alumnos, en la actualidad, ha demostrado necesitar de un más detallado y justo sistema de valoración. Los esquemas con los que hoy trabajan muchos docentes son demasiado mecánicos, imparciales u obtusos. Aun cuando las propuestas teóricas diversifican los recursos y viabilizan la metodología de recogida de datos y emisión de juicios, resulta bastante pertinente profundizar, puntualizar y afinar los subprocesos internos de análisis y valoración de la información para optimizar la concepción y aplicación de los sistemas de evaluación que en la actualidad se ejercen.

El amplio camino recorrido por la definición y desarrollo evaluativo ha forjado diversas formas de interpretar uno de los procesos psicológicos superiores más importantes, el cual se relaciona con la capacidad de juicio que toda persona desarrolla a lo largo de su vida (Frola, 2008). De este modo, “(...) son muchos los esquemas, ordenadores o propuestas que se han formulado para identificar las diferentes etapas o momentos que se han dado en materia de evaluación (...)" (Muñoz, 2007, p. 160). Al respecto, Muñoz reconoce cinco generaciones: desde la medición estandarizada de resultados, hasta la sistematización investigativa de calidad evaluativa.

Teóricos han desarrollado múltiples modelos de evaluación a partir de diferentes funciones. Tal es el caso de la evaluación democrática de MacDonald (1977, citado en Stake, 2004) ${ }^{2}$, quien asume que la evaluación está en función de quién la aplica por cuanto le sirve y cómo utiliza la información recopilada. Esta no se cimienta propiamente en principios democráticos, sino “(...) porque su atención a la experiencia vivida permite documentar la dinámica de la acción humana individual a lo largo del tiempo (...)" (Stake, 2004, p. 280).

La diferenciación de la evaluación comprensiva o interpretativa de la basada en estándares o a partir de criterios de Stake consiste en que la primera “(...) se cimienta sobre el análisis de variables descriptivas (...)" (Stake, 2004, p. 22) y la otra "(...) se desarrolla sobre el conocimiento experiencial y personal en un espacio y un tiempo reales y con personas también reales (...)" (Stake, 2004, p. 22).

La evaluación iluminativa de Parlett y Hamilton, citado en (Mateo, 2000) tiene como objetivo descubrir y documentar a cualquiera que participa en el esquema: “(...) Se centra en el análisis de los procesos de negociación que tienen lugar en el aula. Estos procesos son vivos y cambiantes, y la evaluación será efectiva a partir de la familiarización intensiva con los problemas (...)” (Mateo, 2000, p. 32).

La evaluación participativa “(...) busca la incorporación activa y consciente de los miembros de la organización en el proceso evaluativo" (Rivas y Donovan, 2001, p. 26).

\footnotetext{
MacDonald (citado en Stake, 2004) reconoció que existía la evaluación burocrática, autocrática y democrática. Cada una le asigna al evaluador una función distinta. En la primera este es exclusivamente un "ayudante contratado"; en la segunda un "asesor experto"; y en la tercera un "intermediario de la información".
} 
Herman, Aschbacher y Winters (1992, citados en Díaz Barriga, 2006) propusieron que la evaluación debía valorar legítimamente aprendizajes que se hubieran enriquecido en tareas complejas y activas, a partir de problemas de la vida real, lo que gestó el aprendizaje y enseñanza situados y su evaluación auténtica, es decir, evaluar aprendizajes contextualizados, enfocándose “(...) en el desempeño del aprendiz" [incluyendo] "(...) una diversidad de estrategias de instrucciónevaluación no solo holistas, sino rigurosas” (Díaz Barriga, 2006, p. 127).

En sus inicios, la evaluación planteaba características muy propias de las corrientes pedagógicas de su época. Aun así, existen trabajos previos importantes que dan línea de partida a conceptos generales de evaluación. En estos se sustentan posturas educativas actuales y, con ello, se generan constructos para esbozar una concepción evaluativa de la evolución académicocompetencial que refleje las expectativas pedagógicas actuales.

\section{Antecedentes de la evaluación}

Los procesos evolutivos que ha sufrido la evaluación áulica del progreso de los estudiantes, desde tiempos remotos, ha sido plenamente significativa. En los siglos XVII y XVIII, las formas de evaluación eran meros mecanismos disciplinarios, que pretendían recoger información acerca del proceso cognitivo, pero primordialmente conductual, de los sujetos. Foucault (1975, citado en Larrosa, 1995, pp. 44-45) argumenta que tales mecanismos estaban diseñados “(...) para permitir un control interno, articulado y detallado, que hagan visibles, a quienes están dentro de ella; en términos más generales, una arquitectura que operaría para transformar a los individuos para actuar sobre ellos (...)"; es decir, para influir en su conducta. Este sistema dio origen a la clasificación de los alumnos, a su selección y categorización de acuerdo con sus habilidades y su comportamiento, lo que promovía la imposición de castigos y recompensas. Foucault definió esto como un sistema de observación jerárquica, esto es, un conjunto de técnicas definitivas para llevar a cabo el poder disciplinario (Larrosa, 1995).

Desde luego, ante el sistema educativo institucional existían teóricos que se oponían al proceso pedagógico en general; tales son los casos de Rousseau, Kant, Pestalozzi, entre otros, quienes promulgaban un sistema más armonioso, centrado en las inquietudes y necesidades infantiles y la libertad humana. Para Rousseau (1978, citado en Fernández, 1985, p. 33): “(...) el niño lleva dentro de sí la razón, el deseo de placer y la aversión al dolor (pudiendo reagruparse estos dos últimos en el amor de sí) que, convenientemente manipulados por el preceptor, pueden convertir la educación en un proceso natural, es decir, en algo que surge de las propias necesidades del niño (...)", no de la concepción pura del adulto como un requerimiento deontológico para el infante, ignorando sus propias facultades y deseos. Kant, por su parte, establecía que las actitudes hacia el bien no eran gestadas de manera natural en el ser, debían ser educadas en un marco libre y humano: "El hombre tiene primero que desarrollar sus disposiciones para el bien; la providencia no las ha puesto en él ya terminadas; son meras disposiciones, sin el distintivo de la moralidad" (Kant, 1968, p. 446, citado en Fernández, 1985, p. 34). Pestalozzi (1947, citado en Houssaye, 2003) esclarecía el papel fundamental de la madre en la educación del infante como un motor indispensable previo a la educación formal, en donde el niño desarrollara sus facultades cognitivas y emotivas para su propia tarea educativa. 
Más tarde, con el surgimiento del positivismo, nace la pedagogía positivista: su marco de referencia se centraba en la enseñanza de conocimientos científicos útiles y el desarrollo progresivo del educando. Hoyos (1997) se refiere a la pedagogía positivista -o científica- como un constreñimiento a la epistemología para generar discursos refractarios al sujeto cognoscente y a las relaciones sociales que lo constituyen. Sin embargo, dejó vislumbrar dos aspectos importantes: en primer lugar, recuperó las aportaciones de la filosofía y la pedagogía centrada en el niño y; en segundo, utilizó las pruebas de medición mental para determinar su inteligencia (Hoyos, 1997). Aquí principalmente toman fuerza los exámenes escritos que en el siglo pasado fueron la herramienta universal por excelencia para evaluar el rendimiento académico.

A raíz de esta fuerza coercitiva de examinación, la evaluación se revela como una mera probación del poder del profesor contra la libertad de los alumnos, y ejerce una medida de control disciplinario tanto a la imposición docente como a lo que el profesor espera que el alumno conteste en las pruebas escritas y, en general, a lo que debe saber: lo deseable por el profesor más que por el currículo en sí. Sin embargo, es preciso establecer aquí un punto de bipartición del proceso propio de examinación: efectivamente, por un lado, la aplicación de exámenes ha sido un proceso instrumental que, de manera simultánea, ha acopiado, procesado, interpretado y enjuiciado el progreso educativo sin mayor rigor que su implementación y resolución, e hizo de la evaluación una simple sombra de la valoración, estimación y enjuiciamiento justos y objetivos de un proceso de aprendizaje complejo e integrado; pero, por otro lado, la examinación ha sido el único medio capaz de recopilar información fiable, concreta y específica sobre diversos ejes teóricos, circunstanciales e hipotéticos en asignaturas donde es preciso valorar la adquisición memorística de conceptos, el desarrollo e implementación de algoritmos resolutivos y la solución de problemas concretos, como es el caso de las ciencias duras.

Shepard (2006) realiza una serie de aportaciones destacadas sobre la evaluación áulica formativa a partir de estudios de principios del siglo pasado en los Estados Unidos, en los que cita a teóricos como Thorndike (1913) o Symons (1927), quienes determinaban que la educación podía mejorarse mediante la obtención de pruebas más objetivas. El objeto central consistía en que los docentes, mediante pruebas estandarizadas más apegadas a principios científicos, debían precisar mejor su toma de decisiones. La necesidad de considerar distintos aspectos formativos en el sujeto aprendiz nace de la premisa de que las pruebas objetivas solamente medían "(...) hechos o fragmentos de información en vez de que midieran la capacidad de razonar y la aptitud para la organización (...) (Wood, 1923, citado en Shepard, 2006, p. 10). Tyler (1951, p. 47, citado en Shepard, 2006, p. 11) -considerado por algunos el padre de la evaluación educativa debido a sus postulados modernistasse dio cuenta de que "(...) la medición educativa está concebida, no como un proceso totalmente distinto de la enseñanza, sino más bien como una parte integral [cursivas añadidas] de esta (...)". De tal manera se comienza a vislumbrar la evaluación como un proceso inherente a la intervención didáctica. Aunque en este periodo aún se la concebía como una serie de pruebas y mediciones, la visión sobre su independencia con respecto al proceso de enseñanza había desaparecido y promulgaba una cohesión procedimental a manera de integración al proceso didáctico.

Años después, Nitko (1989, p. 461, citado en Shepard, 2006) promueve una restructuración muy importante de los instrumentos y los procedimientos evaluativos y didácticos; señala que "(la) comprensión que tenga quien diseña pruebas sobre el significado y la estructura del conocimiento que un estudiante trae al sistema de enseñanza' podría utilizarse para identificar 'la comprensión cotidiana de palabras y fenómenos $\{. .$.$\} que no concuerden con la comprensión canónica de los$ 
expertos'". Esta aseveración demuestra la dificultad que existe y ha existido para el diseño de instrumentos fiables de evaluación, simplemente por la codificación de significados entre docentes y estudiantes, además de la poca abstracción del docente hacia la comprensión subjetiva de la misma abstracción natural del alumno. La representación de la realidad docente y la del alumno es totalmente diferente; las comparaciones evaluativas deben ser acordes con su nivel cognitivo -hablamos de experiencia y de nivel de maduración-, este último determinante del grado de pensamiento complejo entre uno y otro, por lo que en el diseño de instrumentos los docentes deben pensar en las condiciones intelectuales de los alumnos.

Shepard (2006) propone que los docentes deben comprobar constantemente el logro de comprensión de sus estudiantes, así como infundirles la importancia de que ellos mismos asuman la responsabilidad de reflexionar y supervisar su propio progreso. También plantea que la evaluación formativa debe atender zonas de desarrollo próximas muy variadas de distintos alumnos, así como diversas formas de andamiaje que van requiriendo estos conforme con sus propios avances; en general, su propuesta de evaluación formativa basada en Sadler (1989, citado en Shepard, 2006), promueve que las progresiones de aprendizaje deben ser el centro de la valoración de los educandos -y no los estándares comunes-, enjuiciados por expertos del currículo disciplinar y basándose en su conocimiento previo; todo ello debe sentar sus criterios valorativos en rúbricas que permitan la verificación objetiva y conjunta, la cual sirva para retroalimentar todo el proceso. Esta enseñanza debe garantizar la transferencia de conocimientos a contextos nuevos y diversos: “(... ¿De qué sirve el conocimiento si no podemos acceder a él o no podemos aplicarlo? (...)" (Shepard, 2006, p. 26).

Las teorías de evaluación actuales demuestran que las nuevas sociedades - del conocimiento y la información-, las condiciones sociopolíticas, los factores de desarrollo natural y las nuevas corrientes pedagógicas deben evaluarse de tal manera que adviertan la complejidad que entraña esa realidad multidimensional y multifactorial, y proponer formas holísticas, éticas y sistemáticas para valorar, estimar y enjuiciar los procesos y los productos evidentes, mediante herramientas y estrategias múltiples acordes con los modelos educativos, campos de estudio y disciplinas científicas, de forma congruente con el desarrollo humano y la prosperidad social ${ }^{3}$, intentando favorecer el desempeño integral de los seres humanos y el desenvolvimiento en su contexto inmediato y mediato, sin olvidar que el centro de la pedagogía actual es el alumno (portador y constructor del saber).

Como hemos mencionado, el proceso de evaluación se centra en la valoración del desarrollo cognitivo de ciertas facultades y capacidades del sujeto aprendiz, pero no exclusivamente en el aprendizaje llano de este -también de las destrezas y habilidades adquiridas, la ejercitación de valores y la concienciación de actitudes y toma de decisiones-; sin embargo, entendemos que es el factor más destacado por evaluar en las condiciones regulares académicas.

La evaluación en el aula -propiamente del progreso académico de los estudiantes- ha hecho notar importantes situaciones que denotan deficiencias dentro de nuestro campo de acción docente y, por amplias referencias, en la educación en general. Dentro de sus mismos subprocesos podemos observar claramente una incongruencia ético-académica, instrumentos con estándares mínimos de

Debe entender el lector por prosperidad social al hecho favorable de las situaciones que acometen la sociedad en su desarrollo evolutivo, desde esta perspectiva, a consecuencia de las acciones educativas. La prosperidad social debe esta enunciada en premisas que procuren el desarrollo científico, tecnológico, artístico y deportivo de una sociedad, integrada por individuos sanos (psicológica y físicamente), cultos, propositivos y proactivos. 
confiabilidad $^{4}$, técnicas y criterios poco ortodoxos, es decir, un sistema de evaluación complicado e ineficiente -tanto para recoger información como para procesarla y emitir juicios- y poco confiable, más aún desde el nuevo enfoque de competencias que plantea exigencias más rigurosas.

\section{El enfoque de competencias y la evaluación}

Este enfoque de competencias, que ha causado un gran revuelo dentro de la educación de esta tercera cultura ${ }^{5}$, puede resumirse como un paradigma curricular que prevé las necesidades educativas contemporáneas cimentadas en el desarrollo pleno de los sujetos, la multiculturalidad, el advenimiento masivo de la tecnología, los cambios climatológicos y sus repercusiones, la deformación sociocultural de los principios de jerarquía adquisitiva de bienes materiales y la carencia evidente de valores del bienestar común. Aunque, sin ánimo de generar polémica, vale la pena señalar que el enfoque es financiado por el Banco Mundial y apoyado por FMI (Fondo Monetario Internacional), el GATT (Acuerdo General de Aranceles y Comercio), el BIP (Banco Internacional de Pagos) y, por supuesto, la UNESCO (Organización de las Naciones Unidas para la Educación, la Ciencia y la Cultura) (Moreno y Soto, 2005). De ello puede inferirse un trasfondo de perpetuación de dominio de clase; no obstante, el enfoque es benévolo con los pedimentos reales de las sociedades contemporáneas y las necesidades didácticas.

Este enfoque está recientemente vinculado de manera muy estrecha con el psicopedagógico socioformativo complejo (ESC), el cual promueve “(...) la formación humana basada en competencias en los diversos contextos, tomando como base la construcción del proyecto ético de vida, las potencialidades de las personas y las expectativas sociales con respecto a la convivencia y la producción (Tobón, 2006, p. 6). Conforme con Tobón (2006, p. 6), todo este entramado está fundamentado en:

(...) el currículo sociocognitivo complejo (Tobón, 2001), la teoría crítica de Habermas (1987), La quinta disciplina (Senge, 1994, 2000), el pensamiento complejo (Morín, 2000a), el paradigma sociocognitivo (Román y Diez, 1994, 2000; Román, 1998, 1999), la formación basada en competencias (Maldonado, 2001), la pedagogía conceptual (Zubiría, 1998) y el aprendizaje estratégico (Pozo y Monereo, 1999) (...).

Además, está fuertemente sustentado igualmente por la teoría de la educación emocional (Salovey y Mayer, $1990^{6}$ y, más tarde, por Goleman, 1995/2004), la teoría de las inteligencias

4 El diseño de los instrumentos de recopilación de información, así como los de verificación, implican un grado de confiabilidad que está en función directa de los objetivos evaluativos, los contenidos revisados, la estructura del instrumento y sus ejes de referencia, su aplicación y supervisión, la interpretación de su información y los criterios de estimación y enjuiciamiento. De este modo, un instrumento será confiable siempre y cuando cada uno de sus componentes mantenga congruencia con los objetivos concretos de evaluación curricular, se apegue a criterios justos que validen su pertinencia, denote una estructura objetiva con reactivos o ejes de atención inteligibles y codificables por sus evaluados, se implemente en condiciones que garanticen el antiplagio, y su sistema de interpretación y enjuiciamiento sea prudente conforme a estándares éticos e imparciales.

5 La primer cultura se refiere al humanismo; la segunda, a la ilustración. La tercera, se entiende desde una nueva filosofía natural fundada en la comprensión de la importancia de la complejidad de la evolución (Brockman,1995, citado en González, 2004).

6 La fuente original de este artículo es Salovey, P. y Mayer, J. D. (1990). Emotional Intelligence. Imagination, Cognition, and Personality, 9(3), 185-211 que aparece compilado en el libro Emotional Intelligence. Key Readings on the Mayer and Salovey Model editado por Peter Salovey y Marc A. Brackett y John D. Mayer; y esta última es la fuente que consulta el autor del artículo. 
múltiples (Gardner, 1983), la enseñanza situada (Brown, Collins y Duguid, 1989), el aprendizaje basado en problemas y las teorías nutrientes del constructivismo en sí (Coll, 1997; Escribano y Del Valle, 20087; Zubiría, 2004).

La metodología para evaluar competencias descansa en la premisa de la valoración y estimación cuantitativa y cualitativa del progreso evidente de competencias diversas según criterios éticos y objetivos. Se fundamenta en la evaluación integral, la cual “(...) debe permitir tomar conciencia frente al desarrollo holístico de todos los procesos que implican: el desarrollo humano, la educación por procesos, la construcción del conocimiento, la transformación socio-cultural desde el liderazgo, y la innovación educativa, pilares esenciales de una Escuela Transformadora y que responden a una propuesta de fundamentos filosóficos, psicológicos, epistemológicos, sociológicos y pedagógicos que asumieron los países cuando decidieron transformar los sistemas educativos y buscar mejor calidad en los mismos" (Iafrancesco, 2005, p. 17-18); en la evaluación holística, a su vez, que busca extraer descripciones a partir de observaciones de lo global y lo concreto, más que de lo simplemente cuantificado y disgregado (Tezanos, Daza y Cabrerizo, 1998). Tobón, Rial, Carretero y García conciben que la evaluación basada desde el enfoque de competencias “(...) se orienta a evaluar las competencias en los estudiantes teniendo como referencia el proceso de desempeño de estos ante actividades y problemas del contexto profesional, social, disciplinar e investigativo, teniendo como referencia evidencias e indicadores, buscando determinar el grado de desarrollo de tales competencias en sus tres dimensiones (afectivo-motivacional, cognoscitiva y actuacional) para brindar retroalimentación en torno a fortalezas y aspectos a mejorar (...)" (2006, p. 133).

\section{Algunas concepciones importantes de evaluación}

Muchos teóricos han dedicado sus investigaciones y propuestas educativas a la evaluación. La concepción general de la evaluación escolar -en todas sus dimensiones, pero primordialmente la de competencias- es relativamente clara en la actualidad. Por un lado denota precisión en su acepción valorativa, aunque, desafortunadamente, por otro, manifiesta insuficiencia en su desarrollo dentro del aula.

Al respecto, Sacristán (1998, p. 337) comenta: “(...) el concepto de evaluación tiene una amplitud variable de significados posibles. Se imponen o no en la práctica según las necesidades a las que sirve la evaluación y en función de las diferentes formas de concebirla (...)”. En este sentido, la evaluación se concibe como un proceso complejo, determinado en la praxis, pero aún así, difícil de concretar, ya que toma diferentes matices de acuerdo con su enfoque, utilidad, propósito y dimensión.

Es necesario hacer esta aclaración debido a que el problema de la evaluación del aprendizaje parte de esta aseveración, es decir, desde la multiconcepción del término educativo y su especificidad limitante. Aunque es muy sabido que la evaluación plantea diferentes dimensiones, propósitos, alcances y, además, puede ser clasificada de muchas maneras, la simple evaluación de los alumnos -sin que esta sea exclusiva del aprendizaje- tiene la característica de ser concebida desde diferentes enfoques. De cualquier modo, resulta conveniente abundar sobre distintas acepciones desde la perspectiva de algunos teóricos educativos clásicos.

El aprendizaje basado en problemas (ABP) fue desarrollado en la Universidad de McMaster en la provincia de Ontario, Canadá en la Facultad de Medicina en 1969 (citado en Escribano y del Valle, 2008). 
Tyler (1950, citado en Tello, 2009, p. 50) asumía la evaluación como el “(...) proceso que determina hasta qué punto se han conseguido los objetivos educativos (...)' " definición que vino bien durante muchos años al enfoque por objetivos que protagonizó el tradicionalismo didáctico.

Más tarde Cronbach (1963:244, citado en Gairín, 1995, p. 60) definió la evaluación como el “(...) proceso de recopilación y utilización de la información para tomar decisiones (...)", idea que, sin duda, marcó la evolución conceptual del proceso evaluativo de una evaluación estática a una evaluación orientativa. Este periodo fue considerado como la primera generación de la evaluación, basada en la medición del contenido curricular, la cual, según Scriven (1967, citado en Tello, 2009, p. 50), se concibe como el “(...) proceso por el que se determina el mérito o valor de alguna cosa (...)".

Scriven subrayó un concepto bastante valioso de la evaluación -aunque por muchos años esto ha generado procesos estimativos tendenciosos-: la meritoriedad. El proceso de valoración y enjuiciamiento educativo es un quehacer de carácter meritorio, en donde los sujetos o entidades evaluadas deben hacer notar el merecimiento de un juicio en consideración de sus facultades evaluadas con justicia, de acuerdo con un método bien definido. Scriven contribuyó a lo que se conoce como la segunda generación de la evaluación.

Bloom y otros (1971, citado en Tello, 2009, p. 50) la proponen como la “(...) reunión sistemática de evidencias a fin de determinar si en realidad se producen ciertos cambios en los alumnos y establecer el grado de cambio en cada estudiante (...)" , percepción, por demás evidente, propia del conductismo pedagógico clásico. Esta, como todos los modelos posteriores a 1967, ya esbozaba, de forma implícita, el enjuiciamiento del evaluador y la tercera generación evaluativa (Muñoz, 2007). Carreño (1985) la entendía como un proceso organizado que permite la obtención de información útil para apoyar la toma de decisiones; una evolución de la concepción crobachiana sobre la evaluación organizacional, que deja ver cierta precisión en la clase de información necesaria para el proceso efectivo.

Nevo (1983) la concibe como la medición de consecuencias deseables e indeseables de las acciones realizadas para alcanzar una meta que se valora. Nevo asumía en su concepción una serie de consecuencias bivalentes, producto de acciones programadas que tenían que corroborarse para estimar, implícitamente, decisiones. Este planteamiento superaba la definición paralizada de determinación de un valor curricular para tomar decisiones; la establecía como un punto alternativo que suponía consecuencias endógenas en la deseabilidad e indeseabilidad, lo que, más tarde, desencadenaría múltiples nuevas concepciones.

Stufflebeam y Shinkfield (1987, p. 19), por su parte, establecieron que la evaluación era “(...) el enjuiciamiento sistemático de la valía o el mérito de un objeto (...)”, definición bastante simple, pero que destacaba el tipo de proceso que la evaluación requería: un proceso sistemático, es decir, un todo compuesto por múltiples elementos que articulada y metodológicamente proporcionan un fin común, validado y completo.

Una definición más actual, precisa y abundante sería la de Ferreres y González (2006, p. 174), quienes (basados en Jiménez, 1997, 1999 y 2002 a y b) la entienden como “(...) un proceso continuo, ordenado y sistemático de recogida de información cuantitativa y cualitativa, que responde a ciertas exigencias -válida, dependiente, fiable, útil, precisa, viable, etc.-, obtenida a través de diversas técnicas e instrumentos, que tras ser cotejada o comparada con criterios establecidos nos permite emitir juicios de valor fundamentados que faciliten la toma de decisiones y que afecten 
el objeto evaluado" ". Los autores precisan diversos elementos que profundizan considerablemente y enriquecen la apreciación de la evaluación como un proceso robusto, sistémico, determinístico y activo. La idea de la negociación implícita en esta concepción define la cuarta generación de la evaluación.

No obstante, es nuestro turno ahora de esclarecer una apreciación de la evaluación escolar de los estudiantes, que se apegue a las necesidades de la sociedad actual; que abarque los aspectos demandados curricularmente; que denote la apertura a los enfoques contemporáneos -primordialmente al de competencias y al socioformativo-, y que retome las características inherentes de la evaluación escolar de manera integral. De tal forma, sería recomendable establecer, además, que la toma de decisiones a la que Ferreres y González se refieren sea para actuar, en consecuencia, a favor del desarrollo del sujeto -en todas sus dimensiones: en el aprendizaje, desarrollo de actitudes y conductas, fomento de valores, estimulación de inteligencias, formación de hábitos, activación de aptitudes, ejercitación de destrezas, etcétera-, ya sea contribuyendo sobre su situación cognitiva o en la metodología didáctica del educador. Además, debemos aclarar que el aprendizaje es simplemente una parte del binomio bidireccional enseñanza-aprendizaje escolar -en donde, efectivamente, el aprendizaje en la actualidad ha demostrado ser autónomo, pero potencialmente óptimo en su andamiaje didáctico- en el cual, tanto docente como discente participan activamente y en el que, como hemos insistido, se denota cierto reduccionismo en los enfoques educativos actuales. Bixio (2003) argumenta su inquietud por mostrar que la evaluación escolar aparece permanentemente centrada en el alumno y que el proceso de enseñanza-aprendizaje es el proceso a evaluar propio dentro de la escuela: “(...) la evaluación forma parte indisoluble de todo el proceso de enseñanza-aprendizaje escolar" (p. 10), sin que esta primera esté circunscrita exclusivamente al proceso binomial, sino inmersa en el complejo progresivo de construir conocimiento y aprehenderlo.

De esta manera, finalmente, la evaluación la entendemos como un proceso de recogida de información, además de continuo, ordenado, sistemático y complejo ${ }^{9}$; que requiere de una dedicada planeación; que es en sí mismo un sistema de subprocesos alternos, de distinto orden pero articulados y que tienen como fin común la constatación y estimación de la construcción del aprendizaje y el desarrollo de facultades del alumno, en donde los resultados arrojados se interpretan con justicia y objetividad para tomar decisiones y actuar, en consecuencia, para incrementar dialécticamente el desarrollo integral de los educandos y su vida plena. Muñoz (2007) lo plantearía como la quinta generación de la evaluación, que implica la investigación social, el enfoque sistémico y su concepción institución-organización per se.

\footnotetext{
Existe una compilación muy completa, como un marco de antecedentes de evaluación educativa y calidad en la educación, llamada "evaluación educativa. Calidad de la educación" de Carlos María de Allende. Es realizado en 1990 por la ANUIES y contiene una bibliografía muy completa y comentada de documentos, artículos y libros con material al respecto; muy valioso para extender nuestro acervo académico y de consulta sobre evaluación educativa en general.

9 La evaluación es considerada como un proceso complejo por su intrincada metodología escolar, mas no por su carácter natural, pues es na actividad humana espontánea. Sin embargo, la evaluación formal ejercida en la escuela conlleva a planificar, confeccionar, desarrollar y valorar los recursos que se llevan a cabo durante todo el proceso educativo, en un comportamiento dialéctico evolutivo. No es nuestra intención abrumar al lector insistiendo en la complejidad de la evaluación como un obstáculo epistemológico de su connotación esencial, sino es preciso concientizarlo de su estructura dinámica y voluminosa, por cuanto es necesario hacer para realizarla de manera justa, sistemática y objetiva.
} 


\section{Aportaciones a la evaluación contemporánea}

Díaz Barriga y Hernández (2002, basadas en Jorba y Casellas, 1997; Miras y Solé, 1990; Santos, 1993; Wolf; 1988), proponen seis aspectos implicados dentro de la evaluación del proceso de enseñanza-aprendizaje:

- La demarcación del objeto, situación o nivel de referencia que se ha de evaluar

- El uso de determinados criterios para la realización de la evaluación

- Una sistematización para la obtención de la información

- Una representación lo más fidedigna posible del objeto de evaluación

- La emisión de juicios

- La toma de decisiones

Aunque Díaz Barriga y Hernández (2002) hacen hincapié en que la sistematización para la obtención de la información debe existir, más no ser rigurosa, nosotros argumentamos que la sistematización debe tener un grado mayor de profundidad y compromiso ético. El diseño eficaz de instrumentos, su selección, técnicas de aplicación y momentos son elementos que determinan significativamente el fracaso o éxito de una evaluación escolar. Además, cabe mencionar que nosotros adoptamos la filosofía administrativa de Nacional Financiera ${ }^{10}$ y otras empresas de vanguardia en el área, en donde la toma de decisiones conduce a acciones, una decisión que no se traduce en acción o acciones en realidad no es una decisión funcional. Díaz Barriga y Hernández, junto con muchos otros teóricos (Livas, 1988; Carreño, 1985; Cronbach, 1963; Ferreres y González, 2006; House, 2000) asumen la implementación de acciones dentro de la toma de decisiones. Consideran que las decisiones tienen una implicación mental y no activa -la intención de distinguir esto es debido a que las decisiones muchas veces quedan estrictamente en nuestra estructura cognitiva, en el escritorio o descritas en algún lugar, sin la determinación o el potencial requerido para implementarlas adecuadamente-.

Stufflebeam, y Shinkfield (1987) y House (2000) insisten en la idea de tomar decisiones al evaluar, asumiendo una elección al decidir, como una acción en sí misma activa, cuando lo ideal es una reacción proactiva. Por tanto, nosotros agregamos un último aspecto que a continuación se explicita:

\section{Implementación de acciones pertinentes proactivas ${ }^{11}$}

De igual modo hacemos hincapié en que la información recopilada es información posible de procesar e interpretar, de acuerdo con ciertos preceptos actitudinales, procedimentales y cognitivos,

10 Nacional Financiera es una empresa que encabeza la política de acción, más que de simple decisión. Las decisiones son elecciones que direccionan el albedrío hacia una preferencia; si una decisión se toma con ligereza, puede solo ser solo un producto idealista y no activo. Esto promueve directamente, como consecuencia obligada, una acción inmediata en la toma de decisiones.

11 Frankl (1946), neurólogo y psiquiatra austriaco, en su libro El hombre en busca de sentido, define la palabra proactividad como una actitud en la que el sujeto asume el pleno control de su conducta vital de modo activo, lo que implica la toma de iniciativa en el desarrollo de acciones creativas y audaces para generar mejoras, haciendo prevalecer la libertad de elección sobre las circunstancias de la vida. El término toma popularidad años después para hacer notar la diferencia entre ser activo y ser dinámico y propositivo en busca de la mejora continua. Al hacer uso del término en la implementación de acciones, nos referimos precisamente al carácter propositivo, eficiente y próspero de las consecuencias deseables de los resultados de la evaluación, primordialmente en los alumnos. 
y los juicios de valor emitidos serán el reflejo del proceso de interpretación, según un enfoque basado en la ética y la pedagogía integral.

Es posible, también, distinguir una dicotomía en la acepción de la evaluación escolar con respecto a su función; Díaz Barriga y Fernández (2002) determina, conforme a Coll (2002), que existen dos funciones inherentes de la evaluación: la función pedagógica y la función social. La primera, se encarga de mediar el proceso de enseñanza-aprendizaje de acuerdo con los resultados esperados y obtenidos, en razón del beneficio cognitivo y desarrollo integral del educando. La segunda, tiene como finalidad la representación cuantificable del desempeño académico, la acreditación, la certificación, la promoción y el reconocimiento de la sociedad. Ambas, de manera conjunta, complementan el sistema evaluador educativo. Es imposible analizar una sin considerar la otra; las dos se entretejen y se permean recíprocamente determinando el progreso escolar de los alumnos y poniéndolo de manifiesto ante la sociedad.

En este sentido, es importante destacar que el concepto y visión de evaluación que un docente asume deviene de la "dimensión psicopedagógica y curricular" (Díaz Barriga y Hernández, 2002), en donde se contempla la conceptualización de la evaluación a partir de un modelo teórico conceptual, las funciones de las tareas de evaluación desde un planteamiento curricular determinado y las decisiones sobre qué, cómo, cuándo y para qué evaluar.

Evaluar a un sujeto es sinónimo de responsabilizarse por lo que se dictaminará como resultado y, por ende, entender que la capacidad o incapacidad de un docente al llevar a cabo la acción evaluadora determinará, de manera significativa, diferentes situaciones del sujeto aprendiz, principalmente, su desarrollo cognitivo (progreso académico), psicosocial (estratificación escolar) y emocional (motivacional).

La incidencia y el entramado de todas las teorías pedagógicas actuales hacen posible la explicación de una necesidad evaluativa efectiva, pertinente y que refiera a los pedimentos sociales en el mundo posmoderno.

La evaluación educativa, en general, nos permite poseer cierto grado de conocimiento acerca de un determinado fenómeno educativo y, con ello, valorar una situación y emitir juicios para actuar. Santos (2000) enfatiza su interés en una evaluación justificada, no tanto en proponer alguna definición que satisfaga las necesidades actuales, sino esclarecer sus razones y propósitos. Al respecto escribe: “(...) lo fundamental, a mi juicio, no es hacer evaluación, ni siquiera hacerla bien. Lo fundamental es conocer el papel que desempeña, la función que cumple, saber quién se beneficia de ella y, en definitiva, al servicio de quién se pone" (p. 9).

Aunque en la actualidad parece indispensable conceptualizar la evaluación educativa, o mejor dicho, definirla considerando un contexto espacial y temporal específico, resulta conveniente, después de haber hecho un esfuerzo por definirla en párrafos anteriores, priorizar sus razones y justificar su importancia.

Fundamentalmente, la inspiración de un sistema de evaluación ${ }^{12}$ reside en su propia epistemología. Las estrategias, instrumentos, criterios, ponderaciones, indicadores, evidencias, momentos, tipos, escalas y demás elementos emergen de la concepción epistemológica y ontológica del proceso complejo que se suscita al recoger, interpretar, valorar y enjuiciar datos, así como en la toma de decisiones y acciones, y no solo en su simple proceder técnico-metodológico.

12 Definimos un sistema de evaluación como el conjunto de elementos que articuladamente ejercen su función específica para que se lleve a cabo un proceo evaluativo. 
Aunque Santos (2000) define su postura de evaluación educativa como una evaluación ideal democrática, en donde todo el contexto, actores y objetos educativos deben ser evaluados por igual; nuestro quehacer epistemológico es concentrarnos en la evaluación meramente del desarrollo integral cognitivo, afectivo, axiológico y volitivo, sin discriminar el vínculo indisoluble que existe en el entramado evaluativo de los demás factores (actores, objetos y contexto) y la última pieza del escalafón del poder.

Él mismo describe propiamente características generales de la evaluación escolar: al alumno se le examina en tiempo y forma determinada; los resultados que recibe son prácticamente inapelables; se le considera fundamentalmente el autor y único responsable de los mismos. Y aunque, a decir verdad, la nueva concepción de la evaluación integral, humanista y constructiva apuntarían a una mayor flexibilidad en tales sentidos, existen factores que no pueden alterarse tan fácilmente. Ejemplo: si las evaluaciones formales u oficiales no tuvieran tiempos específicos bien definidos, los periodos de validación y acreditación correrían riesgos importantes de seguimiento. Ahora bien, en los otros casos podría ocurrir algo distinto. La virtud inapelable del juicio del docente puede ofrecer más democracia según criterios de revaloración, sin que esto signifique restarle autoridad oficial al evaluador, sino, por el contrario, ofrecerle la oportunidad de aceptar su humanidad y subjetividad y, como los jueces de futbol americano, tener la posibilidad de rectificar según otras posturas. Estas otras posturas pueden venir de procesos de valoración conjuntos o individuales -coevaluación y autoevaluación- o, en casos más específicos, de la opinión de otros docentes. De esta manera, el alumno podría adquirir un mayor grado de autonomía y mejor desarrollo de pensamiento reflexivo y crítico, siempre y cuando esto contribuya a fomentar espacios de diálogo y argumentación válidos. De igual forma, catalogar al sujeto evaluado como único responsable de su éxito o fracaso escolar sería demeritar en sí misma la facultad docente y su calidad formadora, así como deslegitimar su función potenciadora. Así, el fracaso escolar en una evaluación no es sinónimo de derrota, es sinónimo de alerta.

Hasta cierto punto es sabido que la evaluación debe ser un proceso sistemático y secuencialmente lógico, en donde sus partes se articulan e integran metodológicamente para un fin claro y preciso. Las bases en donde estos principios se cimienten deben ser justificables en materia pedagógica.

Gronlund (1985) propone algunos principios generales de evaluación:

1. Determinar y aclarar qué es lo que ha de evaluarse.

2. Las técnicas de evaluación deben seleccionarse en términos de los propósitos que han de cumplirse.

3. La evaluación totalmente inclusiva requiere una amplia gama de técnicas de evaluación.

4. La utilización apropiada de las técnicas de evaluación demanda una clara consciencia de sus limitaciones y de sus puntos fuertes.

5. La evaluación es un medio para un fin y no un fin por sí misma.

A decir verdad, aunque para algunos resulten obvias algunas de estas características, es importante hacer notar que para Gronlund, como para muchos otros teóricos, la evaluación requiere de una planificación sumamente meticulosa, independientemente de si los momentos de desarrollo y aplicación están previamente definidos. Cabe aclarar que la evaluación ideal, al igual 
que muchas otras características de la didáctica, deben ser diseñadas por los propios ejecutores. En este marco, los diseñadores de los sistemas evaluativos debieran ser los docentes a cargo de aplicar los modelos, y tendrían la tarea de elaborar y delinear las formas, instrumentos, reactivos, criterios, ponderaciones, momentos, tiempos, indicadores, interpretaciones, valoraciones, calificaciones y, por qué no, sistemas de acreditación de uno o un grupo de alumnos, asistidos en buena medida por los actores directos mismos: los sujetos evaluados. La evaluación actual, esta quinta generación, promueve una evaluación de calidad, asumida como “(...) una decisión personal, para efectuarla según el estilo de vida del actor, quien de acuerdo a su escenario, la convertirá en un ejercicio profesional, en una investigación permanente, en un desempeño de libertad, y sobre todo en una práctica de amor (...)” (Muñoz, 2007, pp. 191-192).

Si bien sabemos que esto es una utopía educativa, al menos debemos participar activamente en el diseño y reajuste de las características de nuestro propio sistema evaluador, lo más estrecho posible al sistema institucional y curricular en el que nos encontremos, de tal manera que nosotros tengamos porcentualmente mayor participación como sujetos evaluadores primarios.

García (1989), por su parte, define igualmente algunos aspectos a considerarse en la evaluación que habremos de tomar en cuenta:

a) holística e integrada

b) contextualizada

c) coherente: epistemológica, que el proyecto educativo se evalúe

d) formativa

e) regida por la ética formal y sustancial

Sin duda los aspectos sugeridos no solo nos obligan a repensar el fin y función de la evaluación necesaria, sino a precisar su especificidad. Por supuesto habrá de estar basada en la compleja realidad de los sujetos y en cada una de sus partes implicadas como un sistema integral; deberá estar cimentada en el contexto que se desee verificar; tendrá que ser pertinente; deberá emitir juicios cuantitativos y cualitativos y; habrá de ser producto de la justicia y objetividad.

Una guía práctica para establecer diez pasos para llevar a cabo una evaluación podría ser la que propone Tenbrink (2006), distribuidos en tres fases: preparación (1 al 5), recogida de datos (6 y 7) y evaluación (8 al 10):

1. Especificar los juicios a emitir y las decisiones a tomar -o posibles decisiones a tomar-

2. Describir la información necesaria

3. Localizar la información ya disponible

4. Decidir cómo y cuándo conseguir la información necesaria

5. Construir o seleccionar los instrumentos de recogida de información

6. Obtener la información necesaria

7. Analizar y registrar información

8. Formular juicios

9. Tomar decisiones e implementar acciones (sugerido por nosotros)

10. Resumir y dar a conocer los resultados de la evaluación 
Tenbrink, en el paso 7, resume un proceso que debiera estar explícito en dos etapas. Primeramente, el analizar la información se establece al disgregar los elementos que la componen con fines de observación, estudio y comprensión; sin embargo, el hecho de comprender la información atomizada requiere de un proceso hermenéutico minucioso, que involucra la lectura atenta, la decodificación y la re-codificación sintética. Implicar la interpretación dentro del análisis resta importancia a su función de vital categoría.

Otro de los factores indispensables en la evaluación es establecer sus funciones. Carreño (1985) señala algunas importantes [cursivas añadidas]:

- Ayudar al que aprende a darse cuenta de cómo debe cambiar o desarrollar su comportamiento (actitud, en este caso)

- Permitir al que aprende a cómo obtener satisfacción cuando está haciendo algo como es debido (motivación)

- Proveer al que aprende una base para las decisiones subsiguientes sobre lo que aprende (saber ser)

- Discernir el procedimiento que convenga seguir (saber decidir)

- Suministrar información necesaria para poder revisar la totalidad del programa de estudio (o en este caso de los temas revisados y los proyectos y problemas abordados)

Lógicamente, además de estas, no podemos olvidar la función primordial de evaluar: obtener información para con ello contribuir al desarrollo mismo del sujeto de manera integral y de sus competencias dentro de una sociedad.

Ahora, las valoraciones en evaluación pueden tener dos tintes: cualificar o cuantificar las interpretaciones. La evaluación puede emitir juicios dialógicos que recuperen situaciones de crecimiento, fortalecimiento, replanteamiento o rectificación, mediante un sistema comunicativo eficaz y asertivo, o bien, plantear todo el complejo epistemológico-metodológico en un valor escalar.

\section{Hacia una nueva evaluación basada en competencias}

Si bien es necesario un modelo que considere ambas posturas, tanto el número como el enunciado interpelado, en la evaluación escolar basada en competencias es preciso apuntalar que los procesos debieran darse como subsistemas no paralelos, es decir, en etapas distintas. Ante la cruda dicotomía clásica del paradigma de investigación cuantitativo y cualitativo, Cook y Reichardt (2005) establecen que en la investigación evaluativa ambos paradigmas pueden coexistir, cada cual con sus características: “(...) la combinación de los dos sería más adecuada para las necesidades de la investigación (...)” (p. 30), y asimismo para la determinación de los resultados de una evaluación.

Dentro de la teoría evaluativa corriente, los resultados juiciosos tienen que obedecer a características peculiares. Deben:

- ser inteligibles y decodificados sin mayor complicación

- revelar con fidelidad la realidad evaluada

- pertenecer a la moralidad y objetividad evaluativa definida 
- contribuir a la reconstrucción de los hechos evaluados con miras de mejorar su composición o acción, y

- estar en un rango socialmente estándar de cualidades o cantidades

Este último aspecto es el que define la manera de cómo describir la realidad evaluada. Un número, por muy preciso en su longitud decimal, solo bosqueja una aproximación tangible que, para fines autómatas -de suficiencia, acreditación o clasificación-, funciona ágilmente. Sin embargo, la realidad compleja no puede evaluarse más de esta forma únicamente; los preceptos verbales, la adjetivación abundante, la construcción de significados holísticos, la interpretación sistemáticohumanística de los progresos y la verbalización pertinente de los procesos y productos evidenciados conforman la otra cara del juicio requerido. Este último, es el más específico y necesario para el enjuiciamiento justo y objetivo.

Los métodos de evaluación tradicionales generalmente carecen de una evaluación cualitativa factual y descrita. La evaluación preponderante en la sociedad actual del conocimiento requiere que los resultados no tan solo se construyan en ambas categorías, sino que exista una metodología clara y escrupulosa para obtenerlos. Tal metodología debe especificar un orden lógico de construcción y, como señalamos antes, deberá ser de manera no paralela, sino una como consecuencia de la otra.

El resultado medible, tangible, es el resultado final y oficial, el que determina la estratificación académica y la clasificación aprobatoria / reprobatoria de un grupo escolar. Este resultado, aunque no es el definitivo en ninguna esfera humana, concentra un peso social descomunal. Tal es el impacto que puede llegar a determinar índices importantes de deserción y rezago educativo o, en otras circunstancias mayores, pero innegables, hasta traumas y trastornos. De este modo, es imprescindible cuidar el aspecto cuantitativo de los resultados para que no se manifieste como un descuido, una malinterpretación, un valor azaroso o un error, pero tampoco como una muestra sobrevalorada o no meritoria del progreso esperado.

Este resultado escalar debe responder a un proceso riguroso de interpretación, pero no tan solo de los productos académicos, ni del mérito resuelto de su cumplimiento escolar o de su comportamiento apegado a determinada disciplina impuesta. Debe ser reflejo estrechamente fidedigno de la interpretación de la realidad escolar integral del alumno.

Entendido esto, debemos establecer que la evaluación cualitativa permite, según Monedero (1998), la reconstrucción y descripción de la realidad. Resultaría prácticamente imposible apegar a consciencia los resultados de tal realidad a un valor medible si nos enfocáramos exclusivamente en interpretarlos y realizar una conversión escalar. Por tanto, lo conveniente indica hacer primeramente una valoración descriptiva, sistemática y meticulosa del complejo escolar del alumno en su realidad ontológica educativa, a partir de su desarrollo integral en consideración de sus facultades cognitivas, afectivas, axiológicas y volitivas.

Así, la evaluación se realizaría, en primera instancia, de manera cualitativa; se agotarían -en relación a lo posible- los medios para interpretar y describir la realidad escolar de los alumnos en pro de su estimulación afectiva y cognitiva y, a partir de ahí, se reinterpretaría tal información considerando la escala valorativa oficial, tomando en cuenta ya el registro continuo de sus productos y procesos dentro de sus ponderaciones e indicadores. Esto no significa, de ningún modo, desvirtuar del proceso mismo, estimando valores de manera casual, tendenciosamente o apegados a una falsa compasión humana, sino tener la apertura a posibilitar, conscientemente y con ética, la estimación 
de los resultados fidedignos que ameriten, conforme a su desarrollo integral, respetando puntos de vista, haciendo hincapié en la subjetividad de las interpretaciones, pero demostrando empatía en las negociaciones y alimentando la motivación.

Precisamente la apreciación cualitativa puede permitir, con mayor claridad, la objetividad de los juicios y la imparcialidad de los criterios resultantes. Ello puede resignificarse en un valor escalar de forma mucho más precisa que la que ofrecen los métodos autómatas simples de conversión directa que los instrumentos de recolección generalmente utilizan. La valoración cualitativa, entonces, recupera la información acopiada, la interpreta y analiza, la enjuicia y registra; luego, se reinterpreta mediante instrumentos de verificación -matrices de rúbricas, hojas de escalas estimativas o listas de cotejo- y se pondera un valor escalar a partir de las conjeturas estimadas.

Cualificar para cuantificar responde a una necesidad propia del modelo psicopedagógico posmoderno, que procura rebasar los resultados del modelo conductista que aún prevalece en las escuelas.

En sí, una evaluación que pretenda ser un proceso integral, que busque la valoración efectiva de los sujetos aprendices en condiciones diversas y que pondere la calidad humana y no tan solo el método intersubjetivo de recepción-acomodación-asimilación de información, no puede ser un proceso exclusivamente técnico-metodológico. Es decir, no puede ser la simple conversión ponderada de los productos y procesos; no puede estar limitada por la consecución de evidencias sujetas a un indicador y a una puntuación; ni tampoco puede ser la acumulación de valores parciales que han representado sucesivamente desempeños diversos o su promedio. La evaluación, en estos términos, debe llegar aún más lejos. Debe contemplar la calidad de una valoración que pormenorice todo ello, pero además se afine en la apreciación del evaluador. Dicha apreciación tendrá que estar sometida a un proceso de análisis y síntesis concienzudo, que otorgue el mérito objetivo de la evolución del desarrollo de todas sus competencias, acorde con una visión holística progresiva de la situación inicial de los educandos y del logro conseguido a lo largo del proceso didáctico. Es cierto que los progresos deben estar en función de los objetivos curriculares -o indicadores de logro-, pero estos no pueden pasar por alto las capacidades innatas individuales. El nivel de logro de los indicadores puede movilizarse de tal forma que responda a expectativas docentes previamente establecidas, siempre y cuando no subvalore los indicadores mínimos de desarrollo y entorpezca los fines educativos grupales y curriculares. Por ello, es preciso cualificar antes de reunir todas la evidencias para cuantificar y emitir resultados terminales, para así poder equiparar y equilibrar ambos aspectos y esto nos conduzca a un resultado final más justo y fidedigno.

Las nuevas tendencias de la evaluación, acordes con las necesidades imperantes de las sociedades actuales, propiamente basadas en el enfoque de competencias, obedecen, en resumen, a lo siguiente:

- Definir desde un inicio las condiciones en las que los sujetos serán evaluados, así como planear detalladamente distintos elementos

- Establecer criterios y ponderaciones en conjunto (docentes y alumnos)

- Diseñar instrumentos variados (ponderando la examinación en contenidos factuales o algorítmicos)

- Formular estrategias de aplicación de pruebas para evitar el plagio, la copia parcial o total de productos

- Basarse en otras opiniones para interpretar datos cualitativos, es decir hacer uso de la coevaluación y la heteroevaluación 
- Valorar y enjuiciar los procesos y productos antes de determinar valores escalares, es decir, cualificar para cuantificar

- Tener la posibilidad de evaluar de manera potencial, lo que significa poder establecer parámetros evaluativos no previstos en las temáticas de clase, pero posiblemente deducibles

- Emitir resultados tanto numéricos como descriptivos

- Utilizar los resultados, principalmente los cualitativos, para replantear el ejercicio didáctico

Finalmente, es necesario recuperar que la evaluación es una mera comparación de esquemas preestablecidos (deseables) con parámetros evidentes y no evidentes, donde los primeros aseguran, de buena o mala manera, un punto de comparación, pero los segundos requerirán de manifestarse en algún tipo de evidencia para poder ser tomados en cuenta. Esta visión, ciertamente reduccionista, permite al evaluador analizar cada elemento, función y fin evaluativo, y de esta manera poder replantear su propio quehacer evaluativo y didáctico y así implementar mejoras a su sistema. Este es un trabajo que el docente debe desarrollar de manera muy cuidadosa y con ello ganar terreno en su acción de interventor didáctico.

\section{Conclusiones}

La evaluación del progreso estudiantil es un proceso meritorio innegable; los alumnos van siendo acreedores de su calificación conforme van desarrollando facultades y estimulando sus capacidades. Desde la visión educativa contemporánea, este hecho tiene lugar en la recuperación de evidencias procesuales y materiales que reflejan la evolución de habilidades, conocimientos, actitudes o valores correspondientes a su desempeño integral. Esta actividad, indudablemente metódica y sistemática, debe ser el producto de un sistema bien diseñado de recolección, interpretación, análisis, valoración y enjuiciamiento de evidencias que revelen de manera fidedigna un juicio justo y objetivo.

La avasallante calificación numérica durante muchos años ha sido un factor representativo descuidado, injusto e impropio. La evaluación contemporánea necesaria, en este sentido, no tan solo requiere de la valoración de competencias diversas sin más: la objetividad y justicia valorativa exigen de un proceso sistemático más escrupuloso y humano, en donde la apreciación integral detallada permita primeramente elaborar juicios adjetivo-descriptivos a conciencia, para luego estar en condiciones óptimas de reinterpretar tales juicios, apoyados de un sistema de ponderación pormenorizado, y estimar un valor escalar que refiera el progreso mayormente apegado a la realidad.

Los subprocesos inmersos en todo esto deben ser diseñados con cautela. La evaluación basada en competencias debe valerse de múltiples recursos para desarrollarse con plenitud y favorecer la recuperación de evidencias y emisión de juicios: implementación de prudentes instrumentos diversificados -no discriminando el examen escrito y utilizando instrumentos tanto de recogida como de verificación de datos (rúbricas, listas de cotejo, etcétera)-, confección de perspicaces estrategias de acopio de información y aplicación de instrumentos, multiapreciación del progreso escolar -hetero, co y autoevaluación-, expresión de juicios verbales constructivos y motivantes, entre otros. La evaluación no es un quehacer sencillo; es un quehacer arduo; pero el más importante para una educación justa y verdaderamente progresiva. 


\section{Referencias}

Bixio, C. (2003). Cómo planificar y evaluar en el aula: propuestas y ejemplos. Argentina: Homo sapiens.

Brown, J. S., Collins, A. y Duguid, P. (Jan-Feb, 1989). Situated Cognition and the Culture of Learning [Cognición situada y la cultura de aprendizaje]. Educational Researcher, 18(1), 32-42. Recuperado de http://www.exploratorium.edu/ifi/resources/museumeducation/situated. html

Carreño, F. (1985). Enfoques y principios teóricos de la evaluación. México: Trillas.

Coll, C. (1997). ¿Qué es el constructivismo? (colección Magisterio 1). Argentina: Magisterio del Río de la Plata.

Cook, T. D. y Reichardt Ch. S. (2005). Métodos cualitativos y cuantitativos en investigación evaluativa (5 ed.). Madrid: Morata

Cronbach, L. J. (1963). Course Improvements Through Evaluation [Curso de mejoras a través de la evaluación]. Teachers College Record, 64(8), 672-683.

Díaz Barriga, F. (2006). Enseñanza situada: Vínculo entre la escuela y la vida. México: McGraw Hill.

Díaz Barriga, F. y Hernández (2002). Estrategias docentes para un aprendizaje significativo. Una interpretación constructivista. México: McGraw-Hill

Escribano, A. y Del Valle, A. (2008). El aprendizaje basado en problemas: Una propuesta metodológica en educación superior. Madrid: Narcea.

Fernández, M. (1985). Trabajo, escuela e ideología. Madrid: Akal.

Ferreres, V. y González A. P. [Eds.]. (2006). Evaluación para la mejora de los centros docentes. Madrid: Praxis.

Frankl, V. E. (1946) El hombre en busca de sentido. Barcelona: Herder.

Frola, P. (2008). Competencias docentes para la evaluación: Diseño de reactivos para evaluar el aprendizaje. México: Trillas

Gairín, J. (1995). Estudio de las necesidades de formación de los equipos directivos de los centros educativos. Madrid: Centro de Publicaciones. 
García, J. M. (1989). Bases pedagógicas de la evaluación. Guía práctica para educadores. Madrid: Síntesis.

Gardner, H. (1983). Frames of Mind: The Theory of Multiple Intelligences [Cuadros de mente: la teoría de inteligencias múltiples]. New York: Basic Books.

Goleman, D. (1995/2004). La inteligencia emocional. México: Vergara. (Trabajo original publicado en 1995)

González, P. (2004). Las nuevas ciencias y las humanidades: De la academia a la policía. México: Anthropos.

Gronlund, N. E. (1985). Measurement and Evaluation in Teaching [Medición y evaluación en la enseñanza]. New York: MacMillan.

House, E. R. (2000). Evaluación, ética y poder. Madrid: Morata.

Houssaye, J. (2003). Cuestiones pedagógicas. México: Siglo XXI.

Hoyos, C. A. (1997). Epistemología y objeto pedagógico ¿Es la pedagogía una ciencia? México: Plaza y Valdés.

Iafrancesco, G. M. (2005). La evaluación integral y del aprendizaje. Fundamentos y estrategias. Bogotá: Magisterio.

Larrosa, J. (1995). Escuela, poder y subjetivación. Madrid: La Piqueta.

Livas, G. (1988). Análisis e interpretación de los resultados de la evaluación educativa. México: Trillas.

Mateo, J. (2000). La evaluación educativa, su práctica y otras metáforas. Horsori: Barcelona.

Moreno, P. y Soto, G. (octubre-diciembre, 2005). Una mirada reflexiva y crítica al enfoque por competencias. Educar, 35, 73-80.

Monedero, J. J. (1998). Bases teóricas de la evaluación educativa. Málaga: Aljibe.

Muñoz, G. A. (2007). Un nuevo paradigma: "La quinta generación de la evaluación". Laurus. Revista de Educación, 13(23), 158-198. http://redalyc.uaemex.mx/pdf/761/76102309.pdf

Nevo, D. (Spring, 1983). The Conceptualization of Educational Evaluation: An Analytical Review of the Literature [La conceptualización de la evaluación educativa: una revisión analítica de la literatura]. Review of Educational Research, 53(1), 117-128. Recuperado de http://rer.sagepub. com/content/53/1.toc 
Rivas, M. T. y Donovan P. (2001). La evaluación participativa (2a ed.). Quito, Ecuador: Avia-Yala.

Sacristán, G. (1998). La evaluación de la enseñanza. En J. G. Sacristán y A. I. Pérez (Auts.). Comprender y transformar la enseñanza (pp. 334-394). Madrid: Morata.

Salovey, P. y Mayer, J. D. (1990). Emotional Intelligence. Imagination, Cognition, and Personality [Inteligencia emocional. Imaginación, cognición y personalidad]. En P. Saovey, M. A. Brackett y J. D. Mayer (eds.). Emotional Intelligence: Key Readings on the Mayer and Salovey model [Inteligencia emocional. Lecturas clave en el modelo de Salovey y Mayer] (pp. 1-30). New York: Yale and New Hampsire Univerities.

Santos, M. Á. (2000). Evaluación educativa 1. Un proceso de diálogo, comprensión y mejora. Argentina: Magisterio del Río de la Plata.

Shepard, L. (2006). La evaluación en el aula. En L. Brennan (Ed.). Educational Mesasurement. (4⿳亠丷厂 ed., pp. 623-646). Colorado: Greengood publishing group. Recuperado de http://www.tec. ac.cr/sitios/Docencia/ceda/Boletin_CEDA/PDF_s/aprendizaje_en_el_aula.pdf

Stake, R. E. (2004). Evaluación comprensiva y evaluación basada en estándares. Barcelona: Graó.

Stufflebeam, D. L. y Shinkfield, A. J. (1987). Evaluación sistemática. Guía teórica y práctica. Barcelona: Paidos.

Tello, I. (2009). Formación a través de Internet: Evaluación de la calidad. Barcelona: UOC.

Tenbrink, T. D. (2006). Evaluación: Guía práctica para profesores. New York: Nárcea. Recuperado de http://books.google.co.cr/books?id=CJyeZusF6YIC\&printsec=frontcover\&hl=es\&source= gbs_ge_summary_r\&cad=0\#v=onepage \&q=describir\%201a\%20información\&f=false

Tezanos, J., Daza, R. y Cabrerizo A. (1998). Propuesta para evaluación y acreditación de programas universitarios en ingeniería. Universidad Boliviana. http://www.ceub.edu.bo/ceub/ secadec/holistica.html

Tobón, S., Rial, A., Carretero, M. Á y García, J. A. (2006). Competencias, calidad y educación superior. Bogotá: Alma mater

Tobón, S. (2006). Formación basada en competencias. Pensamiento complejo, diseño curricular y didáctica ( $2^{\mathrm{a}}$ ed.). Bogotá: Ecoe.

Zubiría, H. D. (2004). El constructivismo en los procesos de enseñanza-aprendizaje en el siglo XXI. Barcelona: Plaza y Valdés. 